\title{
The effect of three different types of walking aids on spatio- temporal gait parameters in community-dwelling older adults
}

\author{
Irene Härdi • Stephanie A. Bridenbaugh • \\ Yves J. Gschwind · Reto W. Kressig
}

Received: 26 September 2012/Accepted: 2 October 2013/Published online: 12 March 2014

(C) Springer International Publishing Switzerland 2014

\begin{abstract}
Background and aims Gait and balance impairments lead to falls and injuries in older people. Walking aids are meant to increase gait safety and prevent falls, yet little is known about how their use alters gait parameters. This study aimed to quantify gait in older adults during walking without and with different walking aids and to compare gait parameters to matched controls.

Methods This retrospective study included 65 older ( $\geq 60$ years) community dwellers who used a cane, crutch or walker and 195 independently mobile-matched controls. Spatio-temporal gait parameters were measured with an electronic walkway system during normal walking.

Results When walking unaided or aided, walking aid users had significantly worse gait than matched controls. Significant differences between the walking aid groups were found for stride time variability (cane vs. walker) in walking unaided only. Gait performances significantly improved when assessed with vs. without the walking aid for the cane (increased stride time and length, decreased cadence and stride length variability), crutch (increased stride time and length, decreased cadence, stride length variability and
\end{abstract}

\footnotetext{
I. Härdi $(\square) \cdot$ S. A. Bridenbaugh

University Center for Medicine of Aging Basel, Basel Mobility Center, Felix Platter Hospital, Schanzenstrasse 55, 4031 Basel, Switzerland

e-mail: Irene.Haerdi@fps-basel.ch
}

Y. J. Gschwind

Falls and Balance Research Group, Neuroscience Research

Australia, Sydney, Australia

R. W. Kressig

University Center for Medicine of Aging Basel, Basel Mobility

Center, Felix Platter Hospital, Burgfelderstrasse 101,

4012 Basel, Switzerland double support) and walker (increased gait speed and stride length, decreased base of support and double support) users. Conclusion Gait in older adults who use a walking aid is more irregular and unstable than gait in independently mobile older adults. Walking aid users have better gait when using their walking aid than when walking without it. The changes in gait were different for the different types of walking aids used. These study results may help better understand gait in older adults and differentiate between pathological gait changes and compensatory gait changes due to the use of a walking aid.

Keywords Mobility aids · Gait · Older adults . Community living

\section{Introduction}

The ability to walk is one of the most relevant and important activities of daily living. It is central to the independence, health and well-being of older people. However, walking may be impaired by many factors such as acute or chronic diseases and physiological changes due to aging $[1,2]$. Impaired gait in older people can negatively affect their functionality, participation in social activities and quality of life [3] and increase their risk of falling [4]. Gait impairment can consequently lead to a loss of independence. The use of a walking aid, such as a cane, crutch or walker, by older people with walking difficulties should help them cope with daily activities and allow them to remain functionally independent and mobile [5, 6]. It seems a surprising paradox then that Rubenstein and Josephson [7] showed that the use of a walking aid was associated with a 2.6-fold (confidence interval 1.2-4.6) increased risk of falling. This may be due to improper use 
of the aid, tripping over the aid or the aid's impediment of compensatory stepping and grasping mechanisms during balance recovery to avoid a fall [8, 9]. However, the increased fall risk associated with the use of a walking aid [7] likely reflects the decreased functional status and mobility of the older people using the walking aids (those with gait disturbances, muscle weakness and increased fall risk are the ones most likely to use a walking aid) rather than due to the walking aid itself. When walking aids are appropriately chosen for clinical conditions (e.g., a standard walker instead of a front-wheeled walker for people with Parkinsonian gait disturbances), correctly sized (not too high or too low) and properly implemented, they can increase walking safety and thus may reduce the risk of falling. These aids can maintain walking ability and improve balance by increasing the base of support, enhancing lateral stability and reducing lower-limb loading [5]. Some older adults comment that they feel steadier and safer when walking with their walking aid [10].

Although walking aids are often used by those with gait impairments, little is known about the effects of different walking aids on gait parameters. Measurements of temporal and spatial gait parameters are used to identify gait deficits and to screen older people for their risk of falling [11]. Impaired gait is characterized by reduced walking speed, shorter stride length and increased gait variability [12, 13]. Small changes in gait variability (stride length variability, stride time variability) have been identified as reliable fall predictors in older adults [13-16]. Furthermore, increased base of support is also predictive of falls [15]. Understanding how gait is influenced by different walking aids may help to distinguish between pathological gait alterations and healthy, compensatory reactions to the use of a walking aid. Although some normative gait data from a selected group of very healthy older adults have been previously described [17], data from population studies are needed to know what "normal" gait is in a representative population of older adults, including those who use walking aids.

The first objective of this study was to investigate whether people over the age of 60 who use a single-tip cane, a forearm crutch or a four-wheeled walker have differences in spatiotemporal gait parameters, while walking normally (selfselected pace) with versus without their usual walking aid. The second objective was to compare these gait parameters to an independently mobile-matched control group. We hypothesized that walking aid users would walk faster, would have a smaller base of support and a decreased stride time variability with their walking aid compared to walking without their walking aid. We also hypothesized that all gait parameters would be more variable in the group of walking aid users when compared to a matched control group.

\section{Methods}

Study design and population

In this retrospective, cross-sectional study, data were collected between January 2007 and October 2011 from patients at the Basel Mobility Center, Felix Platter Hospital, Switzerland. Patients were (1) inpatients at the University Hospital Basel, referred by their attending physician for a gait analysis because of gait disorders and/or falls; (2) community dwellers, referred by their family physician for a gait analysis because of gait disorders and/or falls; (3) older cognitively healthy participants of various studies at the Felix Platter Hospital Memory Clinic or at the Basel Mobility Center; or (4) outpatients from the Felix Platter Hospital Memory Clinic, referred by their family physician because of memory problems. The study was approved by the local ethics committee.

The data used for this study were extracted from an existing database. Inclusion criteria were as follows: (1) Age older than 60 years, (2) community dwelling, (3) use of a walking aid while walking outdoors (those who used a walking aid only indoors were excluded, those who used a walking aid indoors and outdoors or only outdoors were included) for at least 1 month prior to their gait analysis, (4) use of a single-tip cane, forearm crutch or four-wheeled walker, (5) available data for gait analysis with and without walking aid, and (6) Mini Mental State Examination (MMSE) score available. Exclusion criteria were as follows: (1) use of several different walking aids (e.g., cane for shorter distances and walker for longer distances), (2) use of a cane or crutch on both sides (because these subgroups of walking aid users were too small in this study to permit meaningful analysis), (3) improper use of the walking aid (moving cane/crutch with the ipsilateral rather than the contralateral leg; holding the cane or crutch in air while taking multiple steps), (4) severe neurological (normal pressure hydrocephalus, stroke with residual hemiplegia, Parkinson syndromes), orthopedic (severe osteoarthritis of the hips or knees, amputations with or without a prosthesis), or psychiatric illness (major depression, schizophrenia) causing inability to understand or follow task instructions or to walk $15 \mathrm{~m}$ without assistance, (5) terminal illness with life expectancy $<12$ months.

Of the sample of 366 walking aid users, 65 fulfilled the eligibility criteria and were classified into one of three groups according to type of walking aid used: A single-tip cane (hereafter referred to only as cane), a forearm crutch (hereafter referred to only as crutch) or a four-wheeled walker (hereafter referred to only as walker). All walking aids were the personal property of the patients. No alternative walking aids were given and no setting adjustments of the aids were done at the Basel Mobility Center. 
The patients with walking aids were matched to controls from the same database according to gender, age and MMSE.

\section{Gait assessment}

The spatio-temporal parameters of gait were collected with the GAITRite ${ }^{\circledR}$ system (GAITRite ${ }^{\circledR}$ Platinum, CIR System, Sparta, NJ, USA), a 10-m-long electronic walkway with integrated pressure sensors [18]. The walking trials were performed according to the European guidelines for spatiotemporal gait analysis [19]. The standard gait analysis at the Basel Mobility Center consists of five different walking tasks, whereas walking at self-selected normal walking speed (referred to as normal walking in this text) represents the first task. Normal walking with the aid (for those who had them) was performed as an additional sixth task. Details regarding the description of the gait analysis at the Basel Mobility Center have been reported elsewhere [11]. Before testing, a trained evaluator gave standardized verbal instructions regarding the test procedure. In order to measure steady-state gait, the patients initiated and terminated each walk $2 \mathrm{~m}$ before and after the 10-m walkway allowing sufficient distance to accelerate and decelerate. Each patient performed one trial for each of the testing conditions. No practice trials were performed. To ensure safety, patients wore a Posey ${ }^{\circledR}$ safety belt around their waist and were accompanied during each walk by a trained evaluator who walked behind and slightly to the side of the patients. This way, the evaluator could grasp the safety belt if needed, but was outside the range of patient's peripheral vision, to avoid distraction during walking, and next to the electronic walkway, so that only the patient's footfalls were recorded. The patients performed all trials wearing their own footwear and used their own walking aid for the walking aid condition. A video camera was used during the gait analysis to allow detailed review.

Primary outcome measures were the following nine spatio-temporal gait measures: Gait speed $(\mathrm{cm} / \mathrm{s})$, cadence (number of steps per minute), stride time (s), stride time variability $(\%)$, stride length $(\mathrm{cm})$, stride length variability $(\%)$, base of support $(\mathrm{cm})$, base of support variability $(\%)$ and double support (percentage of gait cycle). Variability variables were quantified as the coefficients of variation $(\mathrm{CV}=($ standard deviation/mean $) \times 100)$.

\section{Clinical evaluation and assessments}

Clinical assessment including a physical examination was performed by a physician or a trained research assistant. The following data were used for the present study: age, sex, height, weight, falls (defined as unintentionally coming to rest on the ground or other surface [20]) in the preceding 12 months (single-item question), fear of falling (single-item question, answer graded as yes or no), and relevant medical problems. Cognitive status was evaluated by the score of the Mini Mental State Examination [21], whereby cognitive impairment was defined as a score $<24$ out of a maximum 30 points. Basic mobility was assessed with the Timed Up \& Go Test (TUG) [22], which was performed without using walking aids. The TUG measures the time in seconds that it takes for an individual to rise from a chair with armrests, walk $3 \mathrm{~m}$, turn, walk back and sit down again. Older adults who require $14 \mathrm{~s}$ or longer to complete the task have a high risk for falls [23].

\section{Statistical analysis}

Characteristics of baseline assessment and gait parameters were summarized descriptively using either means and standard deviations or frequencies and percentages, as appropriate. Analyses were performed between the walking aid group and the matched control group and between the subgroups according to the type of walking aid (cane, crutch, walker). Test for normality (Shapiro-Wilk test) and homogeneity of variance (Levene's test) were performed for all outcomes. For characteristics of baseline assessment, either the non-parametric Mann-Whitney $U$ test or the non-parametric Kruskal-Wallis $H$ test were used for continuous variables and either the Chi-square test or Fisher's exact test were used for nominal variables. For all gait parameters, comparisons between the walking aid group and the matched control group were performed using the non-parametric Mann-Whitney $U$ test and between subgroups using the non-parametric Kruskal-Wallis $H$ test. Evaluation of the difference between the normal condition and the walking aid condition within the three subgroups was completed by the Wilcoxon signed rank test. The $p$ values were adjusted for multiple testing using the multiple False Discovery Rate (FDR) procedure. Results were considered significant at a false discovery rate of at most 0.05 . Post hoc tests were completed according to the closure testing principle when a statistically significant global test resulted from the FDR procedure. Analyses were conducted using the SPSS version 19.0 (SPSS, Inc., Chicago, IL) software program for Windows.

\section{Results}

Study population

Of the 65 walking aid users included in this study, 40 used a cane (average age $82.4 \pm 5.7,25$ females), 13 used a crutch (average age $79.4 \pm 6.3,9$ females) and 12 used a walker (average age $84.3 \pm 3.9,8$ females). The patients' duration of walking aid use ranged from 1 month to 
Table 1 Patients' characteristics

\begin{tabular}{|c|c|c|c|}
\hline Variable & $\begin{array}{l}\text { Walking aid } \\
\text { group }(n=65)\end{array}$ & $\begin{array}{l}\text { Matched control } \\
\text { group }(n=195)\end{array}$ & $p$ value \\
\hline Age (years) & $82.1 \pm 5.7$ & $81.9 \pm 5.4$ & 0.766 \\
\hline Gender & & & 1.000 \\
\hline Male (\%) & 35.4 & 35.4 & \\
\hline Female $(\%)$ & 64.6 & 64.6 & \\
\hline $\begin{array}{l}\text { MMSE score } \\
\text { (points) }\end{array}$ & $25.4 \pm 4.4$ & $25.5 \pm 4.2$ & 0.962 \\
\hline $\begin{array}{l}\text { Previous fall in the } \\
\text { last } 12 \text { months }^{\mathrm{a}}\end{array}$ & & & $0.003 *$ \\
\hline None $(\%)$ & 50.0 & 70.9 & \\
\hline 1 or more $(\%)$ & 50.0 & 29.1 & \\
\hline Fear of falling $^{\mathrm{a}}(\%)$ & 30.4 & 10.3 & $<0.001 *$ \\
\hline $\begin{array}{l}\text { Living } \\
\quad \text { arrangement }^{\mathrm{a}}, \%\end{array}$ & & & 0.663 \\
\hline Lives alone & 43.1 & 46.4 & \\
\hline Cohabits & 56.9 & 53.6 & \\
\hline Height $(\mathrm{cm})$ & $164.7 \pm 8.6$ & $164.9 \pm 8.8$ & 0.867 \\
\hline Weight (kg) & $71.9 \pm 13.6$ & $68.5 \pm 12.7$ & 0.104 \\
\hline BMI $\left(\mathrm{kg} / \mathrm{m}^{2}\right)$ & $26.4 \pm 4.1$ & $25.1 \pm 3.9$ & $0.025^{*}$ \\
\hline TUG (s) & $18.2 \pm 6.0$ & $11.6 \pm 3.0$ & $<0.001 *$ \\
\hline
\end{tabular}

Values are mean \pm standard deviation or percentages

MMSE mini mental state examination. TUG Timed Up \& Go Test *Statistically significant

${ }^{\mathrm{a}}$ The number of patients in each group is less than the entire sample due to missing information

15 years, with an average of $2.7 \pm 2.7$ years and a median of 2 years. Eleven patients were not able to recall how long they had been using their walking aid. Patients' characteristics did not differ between the three groups of walking aid users (data not shown). Scores on the MMSE demonstrated that $49(75.4 \%)$ had an MMSE score $\geq 24$ points and $16(24.6 \%)$ had an MMSE score $<24$ points. Performance on the TUG differed between the walking aid users. Those with a cane had the fastest TUG time (average $17.0 \pm 4.4 \mathrm{~s}$ ), followed by the crutch users (average $18.9 \pm 6.1 \mathrm{~s}$ ) and then the walker users (average $21.1 \pm 9.2 \mathrm{~s}$ ). However, the TUG times did not significantly differ between the three groups $(p=0.279)$.

A summary of descriptive data of the entire group of walking aid users and matched controls is shown in Table 1. Compared to the control group, the walking aid group experienced more falls in the previous 12 months ( $p=0.003)$, more often had fear of falling $(p<0.001)$, had a higher BMI $(p=0.025)$, and needed more time to perform the TUG test $(p<0.001)$. Because of missing information for the variables falls, fear of falling and living arrangement, the number of patients in the walking aid group was reduced to $60,56,58$, respectively, and in the matched control group to $189,185,192$, respectively.
Gait measurement

Gait measures from the walking aid group as a whole without and with the use of a walking aid and the matched control group are summarized in Table 2. Gait performance for all investigated parameters was significantly $(p<0.001)$ worse in the walking aid group without the use of the walking aid compared with matched controls. The same result was found for the comparison between the walking aid group with the use of the walking aid and the matched controls $(p<0.001)$.

Description and statistics of gait parameters between the different walking aid users are shown in Table 3. Overall for the walking aid group, gait performance without the use of a walking aid was best in cane users and worst in walker users. Significant group differences were found for stride time variability $(p=0.024)$ between all three groups. Significant post hoc differences were found for stride time variability between cane and walker users $(p=0.023)$. When assessed with the use of their walking aid, no significant results were found between the three groups of walking aid users.

Differences between gait measures with and without the use of the individual walking aid are summarized in Table 4. Comparison of gait speed and stride time variability is presented in Fig. 1a, b. Use of a cane led to significantly improved performance in cadence $(p=0.002)$, stride time $(p=0.004)$, stride length $(p=0.002)$ and stride length variability $(p=0.011)$. Use of a crutch led to significantly improved performances in cadence $(p=0.019)$, stride time $(p=0.019)$, stride length $(p=0.006)$, stride length variability $(p=0.023)$ and double support $(p=0.006)$. Use of a walker led to significantly improved gait performance in gait speed $(p=0.008)$, stride length $(p=0.002)$, base of support $(p=0.006)$ and double support $(p=0.003)$.

\section{Discussion}

To date, no quantitative data exist on the comparison of gait alterations in experienced walking aid users during unassisted walking and during walking with a cane, crutch or walker. Our findings showed that the gait pattern among experienced walking aid users differed clearly from an independently mobile control group and the changes in spatio-temporal gait parameters were different for the different types of walking aids used.

In the present study, patients using walking aids had a significantly slower gait during unassisted or assisted walking compared to a matched control group (74.4 vs. $110.3,78.6$ vs. $110.3 \mathrm{~cm} / \mathrm{s}$, respectively). This indicates that the use of a walking aid is likely a marker for impaired gait and not that the use of a walking aid causes slower 
Table 2 Gait parameters in normal walking without and with walking aid compared to a matched control group

Values are mean \pm standard deviation

$C V$ coefficient of variation

*Statistically significant

${ }^{a}$ Mann-Whitney $U$ test between walking aid group without walking aids vs. matched control group

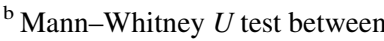
walking aid group with walking aids vs. matched control group

Table 3 Comparison of gait parameters between walking aids in normal walking with and without walking aid
Values are mean \pm standard deviation

$C V$ coefficient of variation

*Statistically significant

${ }^{\text {a }}$ Kruskal-Wallis $H$ test

\begin{tabular}{|c|c|c|c|c|c|}
\hline Gait parameter & $\begin{array}{l}\text { Walking aid group } \\
\text { without aid }(n=65)\end{array}$ & $\begin{array}{l}\text { Walking aid group } \\
\text { with aid }(n=65)\end{array}$ & $\begin{array}{l}\text { Matched control } \\
\text { group }(n=195)\end{array}$ & $p$ value $^{\mathrm{a}}$ & $p$ value $^{\mathrm{b}}$ \\
\hline $\begin{array}{l}\text { Gait speed } \\
(\mathrm{cm} / \mathrm{s})\end{array}$ & $74.4 \pm 19.0$ & $78.6 \pm 17.5$ & $110.3 \pm 20.5$ & $<0.001 *$ & $<0.001^{*}$ \\
\hline $\begin{array}{l}\text { Cadence } \\
\text { (steps/min) }\end{array}$ & $100.3 \pm 11.7$ & $95.4 \pm 10.2$ & $111.2 \pm 10.7$ & $<0.001 *$ & $<0.001^{*}$ \\
\hline Stride time (s) & $1.1 \pm 0.14$ & $1.3 \pm 0.15$ & $1.1 \pm 0.11$ & $<0.001 *$ & $<0.001 *$ \\
\hline $\begin{array}{l}\text { Stride time CV } \\
(\%)\end{array}$ & $3.5 \pm 1.7$ & $2.8 \pm 1.0$ & $2.3 \pm 1.0$ & $<0.001 *$ & $<0.001 *$ \\
\hline $\begin{array}{l}\text { Stride length } \\
(\mathrm{cm})\end{array}$ & $88.9 \pm 18.7$ & $98.7 \pm 17.9$ & $118.9 \pm 17.1$ & $<0.001 *$ & $<0.001 *$ \\
\hline $\begin{array}{l}\text { Stride length CV } \\
(\%)\end{array}$ & $5.3 \pm 3.0$ & $3.9 \pm 1.7$ & $3.0 \pm 1.5$ & $<0.001 *$ & $<0.001 *$ \\
\hline $\begin{array}{l}\text { Base of support } \\
(\mathrm{cm})\end{array}$ & $11.9 \pm 4.6$ & $10.5 \pm 4.0$ & $8.7 \pm 3.3$ & $<0.001^{*}$ & $<0.001 *$ \\
\hline $\begin{array}{l}\text { Base of support } \\
\text { CV (\%) }\end{array}$ & $24.6 \pm 31.3$ & $19.7 \pm 17.5$ & $29.8 \pm 25.3$ & $<0.001 *$ & $<0.001 *$ \\
\hline $\begin{array}{l}\text { Double support, } \\
\% \text { of gait cycle }\end{array}$ & $36.4 \pm 6.7$ & $34.0 \pm 5.2$ & $29.1 \pm 2.7$ & $<0.001 *$ & $<0.001^{*}$ \\
\hline
\end{tabular}

\begin{tabular}{lcccc}
\hline Gait parameter & Cane $(n=40)$ & Crutch $(n=13)$ & Walker $(n=12)$ & $p$ value $^{\mathrm{a}}$ \\
\hline Without walking aid & & & & \\
Gait speed (cm/s) & $77.6 \pm 18.6$ & $70.6 \pm 20.5$ & $68.2 \pm 18.0$ & 0.299 \\
Cadence (steps/min) & $100.3 \pm 11.5$ & $98.7 \pm 10.4$ & $101.95 \pm 14.4$ & 0.908 \\
Stride time (s) & $1.2 \pm 0.15$ & $1.2 \pm 0.13$ & $1.2 \pm 0.15$ & 0.939 \\
Stride time CV (\%) & $3.0 \pm 1.3$ & $4.1 \pm 1.8$ & $4.4 \pm 2.3$ & $0.024^{*}$ \\
Stride length (cm) & $92.6 \pm 18.1$ & $85.5 \pm 19.4$ & $80.4 \pm 18.1$ & 0.162 \\
Stride length CV (\%) & $4.7 \pm 2.0$ & $5.3 \pm 2.9$ & $7.2 \pm 4.7$ & 0.354 \\
Base of support (cm) & $11.3 \pm 3.9$ & $12.2 \pm 6.1$ & $13.6 \pm 4.9$ & 0.225 \\
Base of support CV, (\%) & $23.3 \pm 25.2$ & $26.0 \pm 33.9$ & $27.5 \pm 46.6$ & 0.587 \\
Double support, \% of gait cycle & $34.7 \pm 6.1$ & $38.4 \pm 6.9$ & $39.9 \pm 6.8$ & 0.058 \\
With walking aid & & & & \\
Gait speed (cm/s) & $78.7 \pm 17.8$ & $74.7 \pm 16.9$ & $82.7 \pm 18.0$ & 0.355 \\
Cadence (steps/min) & $95.5 \pm 9.7$ & $92.0 \pm 9.5$ & $98.5 \pm 12.5$ & 0.314 \\
Stride time (s) & $1.3 \pm 0.13$ & $1.3 \pm 0.14$ & $1.2 \pm 0.17$ & 0.303 \\
Stride time CV (\%) & $2.8 \pm 0.9$ & $3.0 \pm 1.3$ & $2.7 \pm 0.9$ & 0.662 \\
Stride length (cm) & $98.8 \pm 18.9$ & $96.7 \pm 15.9$ & $100.7 \pm 17.6$ & 0.699 \\
Stride length CV (\%) & $3.9 \pm 1.5$ & $3.8 \pm 1.8$ & $4.1 \pm 2.0$ & 0.930 \\
Base of support (cm) & $10.7 \pm 3.7$ & $10.5 \pm 5.2$ & $9.6 \pm 3.4$ & 0.338 \\
Base of support CV (\%) & $21.2 \pm 18.0$ & $22.0 \pm 21.5$ & $12.1 \pm 6.5$ & 0.151 \\
Double support, \% of gait cycle & $33.4 \pm 4.9$ & $35.7 \pm 6.3$ & $34.2 \pm 4.7$ & 0.650 \\
\hline
\end{tabular}

gait. In addition to gait speed, all investigated gait parameters, were significantly better in the control group than the walking aid group. Particularly gait variability is known as a marker for irregular and unstable gait and is associated with frailty [24] and an increased fall risk [1315]. Studies comparing gait parameters between fallers and non-fallers among older people have revealed that fallers have slower gait speed, increased double support, stride length variability and stride time variability [13-15]. These findings are in accordance with our present study suggesting that walking aid users might have a greater likelihood of falling than independently mobile older people.

This study demonstrated that there were only discrete differences in spatio-temporal gait parameters among the three groups of walking aid user (cane, crutch, walker). These findings are in contrast to our expectations. Since the 
Table 4 Gait measures of differences between test results without and with walking aid deviation

$C V$ coefficient of variation

*Statistically significant

${ }^{\text {a }}$ Wilcoxon signed rank test

\begin{tabular}{lclcccc}
\hline Gait parameter & $\begin{array}{l}\text { Cane } \\
(n=40)\end{array}$ & $p$ value & $\begin{array}{l}\text { Crutch } \\
(n=13)\end{array}$ & $p$ value & $\begin{array}{l}\text { Walker } \\
(n=12)\end{array}$ & $p$ value $^{\mathrm{a}}$ \\
\hline Gait speed (cm/s) & $+1.1 \pm 14.2$ & 0.707 & $+4.0 \pm 12.1$ & 0.346 & $+14.5 \pm 10.6$ & $0.008^{*}$ \\
Cadence (steps/min) & $-4.8 \pm 9.0$ & $0.002^{*}$ & $-6.7 \pm 8.4$ & $0.019^{*}$ & $-3.4 \pm 12.2$ & 0.594 \\
Stride time (s) & $+0.06 \pm 0.12$ & $0.004^{*}$ & $+0.089 \pm 0.11$ & $0.019^{*}$ & $+0.042 \pm 0.12$ & 0.410 \\
Stride time CV (\%) & $-0.16 \pm 1.4$ & 0.707 & $-1.1 \pm 1.4$ & 0.033 & $-1.7 \pm 2.6$ & 0.050 \\
Stride length (cm) & $+6.2 \pm 11.2$ & $0.002^{*}$ & $+11.2 \pm 9.9$ & $0.006^{*}$ & $+20.3 \pm 9.4$ & $0.002^{*}$ \\
Stride length CV (\%) & $-0.76 \pm 1.6$ & $0.011^{*}$ & $-1.5 \pm 2.3$ & $0.023^{*}$ & $-3.2 \pm 4.0$ & 0.034 \\
Base of support (cm) & $-0.52 \pm 1.8$ & 0.104 & $-1.7 \pm 2.7$ & 0.221 & $-4.0 \pm 3.1$ & $0.006^{*}$ \\
Base of support CV (\%) & $-2.1 \pm 17.3$ & 0.502 & $-4.0 \pm 15.1$ & 0.421 & $-15.3 \pm 41.6$ & 0.182 \\
Double support, \% of & $-1.36 \pm 3.3$ & 0.027 & $-2.7 \pm 2.8$ & $0.006^{*}$ & $-5.7 \pm 4.1$ & $0.003^{*}$ \\
gait cycle & & & & & & \\
\hline
\end{tabular}
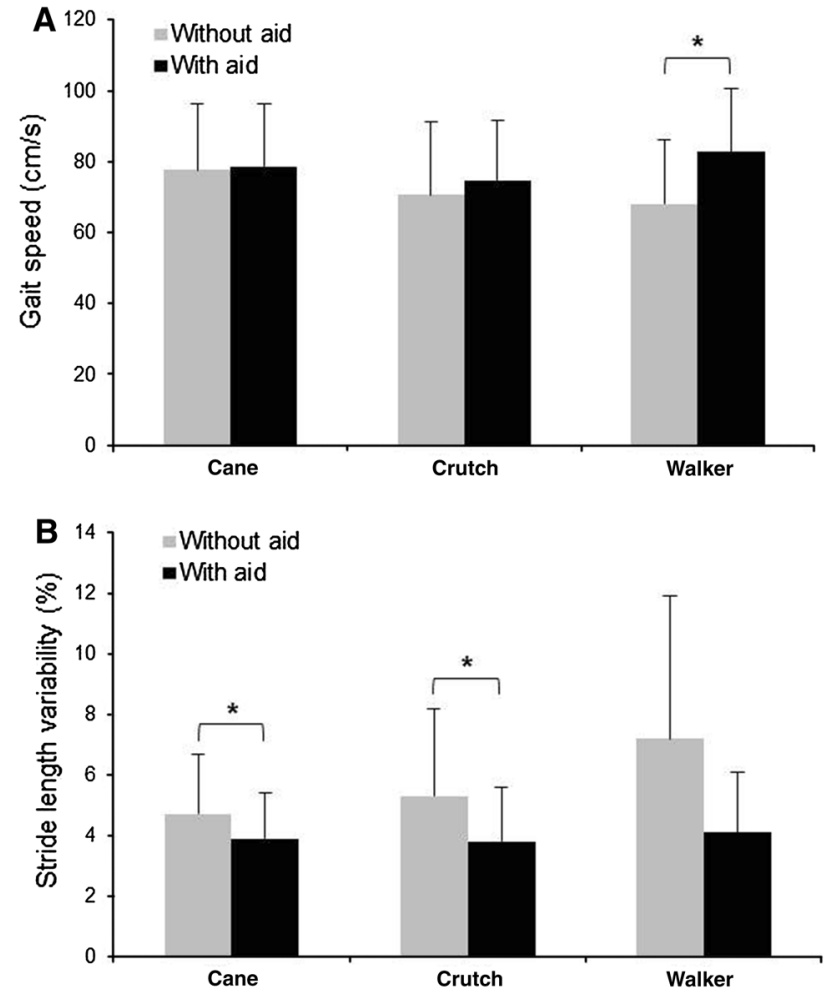

Fig. 1 Gait speed (a) and stride length variability (b) for walking with vs. without walking aid by walking aid group. *denotes significant differences within a walking aid group

selection of the walking aid depends on the individual's functional level and the support of the device increases from cane to crutch to walker, it was hypothesized that cane users would show a better gait pattern than crutch users and that they in turn would perform better than walker users. Although differences in spatio-temporal gait parameters while walking unassisted were relatively discrete, the gait pattern of the cane users tended to be better than the gait pattern of crutch users and walker users with a significant difference in stride time variability between cane and walker users. Interestingly, neither a significant difference in one of the investigated gait parameters nor a tendency between the three groups during assisted walking was detected. From a clinical point of view, it would be of great interest to investigate if an initial objective gait analysis would help in the selection of an appropriate walking aid.

Within each subgroup of walking aid users, gait performance was significantly better in several gait parameters when assessed during walking with the walking aid compared to walking unaided. Using a walker had the greatest effect on gait speed with an increase of $14.5 \mathrm{~cm} / \mathrm{s}$. Using a cane or crutch did not significantly influence gait speed. Verghese et al. [13] reported that each $10 \mathrm{~cm} / \mathrm{s}$ decrease in gait speed was associated with a $7 \%$ increased risk for falls. Based on the results in gait speed, it could be concluded that only the use of a walker provides a benefit. However, one should be aware that walking speed may be affected by other gait parameters. The increase in gait speed among the walker group arising from increased stride length could not be observed in the cane and crutch group due to the simultaneous reduction in cadence. Another explanation for the increase in gait speed may be that the use of a cane or crutch requires the user to lift the device in time with their stepping, whereas the walker allows the person to push the device without lifting it. However, other gait parameters in addition to gait speed are needed to fully understand gait stability in regard to falls prevention.

The use of a mobility aid enlarges a person's supporting area (not to be confused with the base of support gait parameter; see Fig. 2), thereby improving balance [5]. In this context, Bateni and Maki [5] suggest that a walker enlarges the supporting area more than a cane, and therefore the potential to increase stability is even greater. Considering the base of support measured by the GAITRite system, patients within the walker group had a $4 \mathrm{~cm}$ decrease in their base of support during assisted walking. Maki [15] reported that a $4 \mathrm{~cm}$ increase in the base of support of was associated with an increased risk for future falls. Hence, changes in gait 


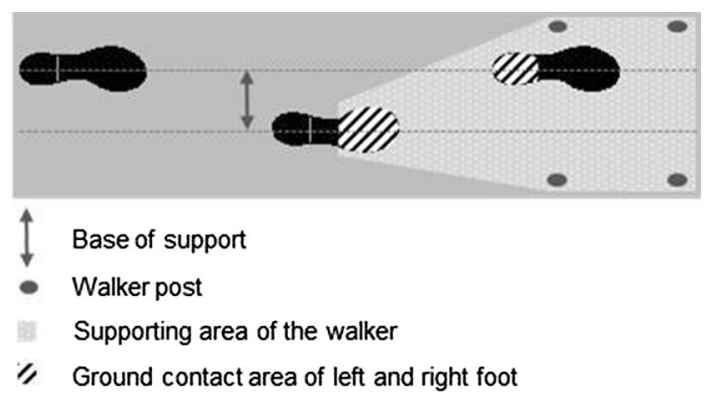

Fig. 2 Schematic illustration of the base of support as measured by GAITRite ${ }^{\circledR}$ and of the supporting area of a walker. Figure modified from Bateni and Maki [5]

parameters seen in our study, particularly decreased base of support and double support, as well as increased stride length indicates that patients adopted a more stable gait pattern when using their walking aid.

Another marker of gait stability is the stride-to-stride variability, which can be viewed as a marker of gait regularity. High stride-to-stride variability reflects gait instability [15]. Our findings show that walking with a cane or crutch significantly decreased stride length variability. Stride time variability, another investigated parameter that reflects stride-to-stride variability, also decreased in all three groups but did not reach statistical significance. Previous studies have been shown that higher variability in stride time and stride length is associated with increased falls in older adults $[13,15,16]$. Hence, utilizing a cane or crutch would indicate a lower fall risk in these two groups of experienced walking aid users when walking with their device than without it.

The ability to safely and effectively use a walking aid while walking characterizes an additional attentiondemanding task. Thus, it could be speculated that the attentional demands associated with the use of a walking aid could well lead to an altered gait performance. A way to reveal the attentional demands of using a walking aid is the comparison of gait performance between experienced walking aid users and first-time users. Studies investigating the effect of walking aids on gait in first-time users showed that gait performance remained unchanged or even worsened [25-28]. In contrast, studies with experienced walking aid users demonstrated an improvement in gait performance $[29,30]$, similar to the results in the present study. Due to the wide variety of methodological procedures and study populations, a final conclusion to guide clinical practice needs more research in this area. However, it is of utmost importance to provide prior training before the use of walking aids and to re-evaluate its need after a certain time span to guarantee the safe and effective use of the device.

When interpreting the results, a few factors need to be taken into consideration. First, the number of patients in the crutch and walker group was small compared to the cane group, which might have influenced study results. However, the statistical procedures used considered the different sample sizes and the distribution of the gait variables. In addition, patients with a range of mobility and health condition were included. Unfortunately, health status was not consistently measured and therefore could not be classified. Finally, the reason for use of the chosen walking aid was not part of the standard assessment, which means little or nothing was known about the actual need for such devices. Future studies should also include standardized measures of health status and comorbidities as well as the reason for use and compare the effect of walking aids among different patient groups according to health status, reason for using or cognitive status.

\section{Conclusion}

This study is the first to report on the effect of different types of walking aids on quantitatively measured gait parameters in community-dwelling older people. The findings of the present study demonstrate that walking aid users have better gait when using their walking aid than when walking without it. The changes in gait were different for the different types of walking aids used. However, the walking aid group had a more irregular and unstable gait performance, e.g., higher stride time variability and stride length variability, than the matched control group. Therefore, using a walking aid improved gait performance but not to a degree that it can be called a healthy, stable gait pattern. Therefore, the selection of a walking aid should depend on objective gait assessments and periodical re-evaluation to ensure that it suits a person's functional requirements and physical capabilities. Furthermore, the results suggest that these changes are a compensatory reaction to the use of the walking aid rather than an indication of a pathological gait pattern in this group of experienced older walking aid users.

Acknowledgments The authors would like to thank Prof. Dr. Manfred Berres for the advice in statistical analyses. We also thank Dr. Katrin Singler for helpful ideas on initial research questions for this analysis.

Conflict of interest None.

\section{References}

1. Sudarsky L (1990) Geriatrics: gait disorders in the elderly. N Engl J Med 322(20):1441-1446

2. Verghese J, LeValley A, Hall CB, Katz MJ, Ambrose AF, Lipton RB (2006) Epidemiology of gait disorders in community-residing older adults. J Am Geriatr Soc 54(2):255-261

3. Metz DH (2000) Mobility of older people and their quality of life. Transp Policy 7(2):149-152 
4. Rubenstein LZ, Josephson KR (2006) Falls and their prevention in elderly people: what does the evidence show? Med Clin North Am 90(5):807-824

5. Bateni H, Maki BE (2005) Assistive devices for balance and mobility: benefits, demands, and adverse consequences. Arch Phys Med Rehabil 86(1):134-145

6. Hartke RJ, Prohaska TR, Furner SE (1998) Older adults and assistive devices: use, multiple-device use, and need. J Aging Health 10(1):99-116

7. Rubenstein LZ, Josephson KR (2002) The epidemiology of falls and syncope. Clin Geriatr Med 18(2):141-158

8. Bateni H, Heung E, Zettel J, McLlroy WE, Maki BE (2004) Can use of walkers or canes impede lateral compensatory stepping movements? Gait Posture 20(1):74-83

9. Bateni H, Zecevic A, Mcllroy WE, Maki BE (2004) Resolving conflicts in task demands during balance recovery: does holding an object inhibit compensatory grasping? Exp Brain Res 157(1):49-58

10. Aminzadeh F, Edwards N (1998) Exploring seniors' views on the use of assistive devices in fall prevention. Public Health Nurs 15(4):297-304

11. Bridenbaugh SA, Kressig RW (2011) Laboratory review: the role of gait analysis in seniors' mobility and fall prevention. Gerontology 57(3):256-264

12. Menz HB, Lord SR, Fitzpatrick RC (2003) Age-related differences in walking stability. Age Ageing 32(2):137-142

13. Verghese J, Holtzer R, Lipton RB, Wang C (2009) Quantitative gait markers and incident fall risk in older adults. J Gerontol A Biol Sci Med Sci 64(8):896-901

14. Hausdorff JM, Edelberg HK, Mitchell SL, Goldberger AL, Wei JY (1997) Increased gait unsteadiness in community-dwelling elderly fallers. Arch Phys Med Rehabil 78(3):278-283

15. Maki BE (1997) Gait changes in older adults: predictors of falls or indicators of fear. J Am Geriatr Soc 45(3):313-320

16. Hausdorff JM, Rios DA, Edelberg HK (2001) Gait variability and fall risk in community-living older adults: a 1-year prospective study. Arch Phys Med Rehabil 82(8):1050-1056

17. Hollman JH, McDade EM, Petersen RC (2011) Normative spatiotemporal gait parameters in older adults. Gait Posture 34(1):111-118

18. Bilney B, Morris M, Webster K (2003) Concurrent related validity of the GAITRite walkway system for quantification of the spatial and temporal parameters of gait. Gait Posture 17(1):68-74

19. Kressig RW, Beauchet O (2006) Guidelines for clinical applications of spatio-temporal gait analysis in older adults. Aging Clin Exp Res 18(2):174-176

20. Lamb SE, Jørstad-Stein EC, Hauer K, Becker C (2005) Development of a common outcome data set for fall injury prevention trials: the Prevention of Falls Network Europe consensus. J Am Geriatr Soc 53(9):1618-1622

21. Folstein MF, Folstein SE, McHugh PR (1975) "Mini-mental state". A practical method for grading the cognitive state of patients for the clinician. J Psychiatr Res 12(3):189-198

22. Podsiadlo D, Richardson S (1991) The timed "Up \& Go": a test of basic functional mobility for frail elderly persons. J Am Geriatr Soc 39(2):142-148

23. Shumway-Cook A, Brauer S, Woollacott M (2000) Predicting the probability for falls in community-dwelling older adults using the Timed Up \& Go Test. Phys Ther 80(9):896-903

24. Montero-Odasso M, Muir SW, Hall M et al (2011) Gait variability is associated with frailty in community-dwelling older adults. J Gerontol A Biol Sci Med Sci 66(5):568-576

25. Bryant MS, Pourmoghaddam A, Thrasher A (2012) Gait changes with walking devices in persons with Parkinson's disease. Disabil Rehabil Assist Technol 7(2):149-152

26. Kloos AD, Kegelmeyer DA, White SE, Kostyk SK (2012) The impact of different types of assistive devices on gait measures and safety in Huntington's disease. PLoS One 7(2):e30903

27. Liu HH, McGee M, Wang W, Persson M (2009) Comparison of gait characteristics between older rolling walker users and older potential walker users. Arch Gerontol Geriatr 48(3):276-280

28. Protas EJ, Raines ML, Tissier S (2007) Comparison of spatiotemporal and energy cost of the use of 3 different walkers and unassisted walking in older adults. Arch Phys Med Rehabil 88(6):768-773

29. Polese JC, Teixeira-Salmela LF, Nascimento LR et al (2012) The effects of walking sticks on gait kinematics and kinetics with chronic stroke survivors. Clin Biomech 27(2):131-137

30. Schwenk M, Schmidt M, Pfisterer M, Oster P, Hauer K (2011) Rollator use adversely impacts on assessment of gait and mobility during geriatric rehabilitation. J Rehabil Med 43(5):424-429 